\section{The human initiator is a distinct and abundant element that is precisely positioned in focused core promoters}

\author{
Long Vo ngoc, California Jack Cassidy, \\ Cassidy Yunjing Huang, Sascha H.C. Duttke, \\ and James T. Kadonaga
}

\begin{abstract}
Section of Molecular Biology, University of California at San Diego, La Jolla, California 92093, USA
\end{abstract}

DNA sequence signals in the core promoter, such as the initiator (Inr), direct transcription initiation by RNA polymerase II. Here we show that the human Inr has the consensus of $\mathrm{BBCA}_{+1} \mathrm{BW}$ at focused promoters in which transcription initiates at a single site or a narrow cluster of sites. The analysis of 7678 focused transcription start sites revealed $40 \%$ with a perfect match to the Inr and $16 \%$ with a single mismatch outside of the $\mathrm{CA}_{+1}$ core. TATA-like sequences are underrepresented in Inr promoters. This consensus is a key component of the DNA sequence rules that specify transcription initiation in humans.

Supplemental material is available for this article.

Received November 14, 2016; revised version accepted December 19, 2016.

The multifarious signals that lead to the initiation of transcription ultimately converge at the core promoter, which is sometimes referred to as the gateway to transcription (for reviews, see Smale and Kadonaga 2003; Goodrich and Tjian 2010; Kadonaga 2012; Danino et al. 2015). The core promoter is the stretch of DNA-which typically is from about -40 to +40 nucleotides $(\mathrm{nt})$ relative to the +1 transcription start site (TSS) - that directs the initiation of transcription. Core promoters are diverse in terms of their composition and function, and their activities are driven by the presence or absence of DNA sequence motifs such as the TATA box, initiator (Inr), TFIIB recognition elements $\left(\mathrm{BRE}^{\mathrm{u}}\right.$ and $\left.\mathrm{BRE}^{\mathrm{d}}\right)$, polypyrimidine initiator (TCT), motif ten element (MTE), and downstream core promoter element (DPE). There are no universal core promoter motifs. Specific core promoter elements can be important for enhancer-promoter specificity (for example, see Butler and Kadonaga 2001; Juven-Gershon et al. 2008) as well as the regulation of gene networks (for example, see Juven-Gershon et al. 2008; Parry et al. 2010; Duttke et al. 2014; Wang et al. 2014).

The long-term goal of this study is to gain a more specific understanding of the human core promoter. It has been estimated, for instance, that $<25 \%$ of human core promot-

[Keywords: RNA polymerase II; initiator; core promoter; transcription start site; focused transcription] Corresponding author: jkadonaga@ucsd.edu

Article published online ahead of print. Article and publication date are online at http://www.genesdev.org/cgi/doi/10.1101/gad.293837.116. ers contain the well-known TATA box or a TATA-like sequence (Gershenzon and Ioshikhes 2005; Carninci et al. 2006; Yang et al. 2007). In fact, it appears that the Inr is the most common core promoter element in humans. For example, $48 \%-49 \%$ of human promoters were found to have a sequence in the TSS region (from -5 to +6 relative to the +1 TSS) that is related to the 8-nt "cap signal" (i.e., Inr) position-weight matrix (based on 502 eukaryotic promoters) (Bucher 1990; Gershenzon and Ioshikhes 2005). In addition, it has been found that $46 \%$ of human promoters contain the $\mathrm{YYA}_{+1} \mathrm{NWYY}$ Inr consensus within -80 to $+80 \mathrm{nt}$ relative to the TSS (Yang et al. 2007). These observations were interesting, but the precise sequence, abundance, and positioning of the human Inr remained to be determined.

The Inr is an extensively studied core promoter element. The presence of a distinct sequence motif that encompasses the TSS was initially described by Corden et al. (1980), and the function of this sequence, which was termed the "initiator," was incisively articulated by Smale and Baltimore (1989). Biochemical studies revealed that the Inr is recognized by the TAF1 and TAF2 subunits of TFIID (Kaufmann and Smale 1994; Purnell et al. 1994; Verrijzer et al. 1995; Chalkley and Verrijzer 1999). The mutational analysis of the human Inr led to the widely used functional Inr consensus of YYA ${ }_{+1}$ NWYY (Javahery et al. 1994; Lo and Smale 1996). However, the genomewide mapping of the $5^{\prime}$ ends of steady-state transcripts by the cap analysis gene expression (CAGE) method yielded the human Inr consensus of $\mathrm{YR}_{+1}$ (Carninci et al. 2006; Frith et al. 2008), which is also commonly used. Hence, the nature of the human Inr is unresolved.

We therefore sought to investigate the human Inr consensus. It is important to have the most accurate as possible representation of the Inr consensus for further studies of transcriptional regulation in humans. This is essential for not only the analysis of the Inr itself but also the identification and analysis of other core promoter elements that act in conjunction with the Inr. Recent advances have enabled the genome-wide mapping of the $5^{\prime}$ ends of nascent transcripts and have thus provided the opportunity to obtain new insights into TSSs and core promoters in humans. In this context, we examined the consensus, occurrence, and characteristics of the human Inr at focused promoters in which transcription initiates at a single site or in a narrow cluster of sites.

\section{Results and Discussion}

Identification of focused TSSs in human MCF-7 cells with FocusTSS

To investigate the human Inr, we sought to generate a data set of focused TSSs that represent specifically positioned RNA polymerase II transcription preinitiation complexes (PICs). We therefore generated two independent 5'-GROseq $\left(5^{\prime}\right.$ end-selected global run on followed by sequencing)

(C) 2017 Vo ngoc et al. This article is distributed exclusively by Cold Spring Harbor Laboratory Press for the first six months after the full-issue publication date (see http://genesdev.cshlp.org/site/misc/terms.xhtml). After six months, it is available under a Creative Commons License (Attribution-NonCommercial 4.0 International), as described at http://creativecommons.org/licenses/by-nc/4.0/. 
(Lam et al. 2013) libraries with human MCF-7 breast carcinoma cells. The 5'-GRO-seq method detects the $5^{\prime}$ ends of nascent transcripts and is related to GRO-cap (Kruesi et al. 2013; Core et al. 2014). These methods capture minimally processed nascent transcripts and are thus well suited for the mapping of the $5^{\prime}$ ends of transcripts.

To identify TSSs, we developed a peak-calling algorithm, termed FocusTSS, which is based on the properties of the PIC. After assembly of the PIC at the promoter, the RNA polymerase II can initiate transcription at a single site or in a narrow cluster of sites (see, e.g., Kadonaga 1990). We thus designed FocusTSS to reflect this property of the PIC. As outlined in Figure 1A, it initially identifies

A

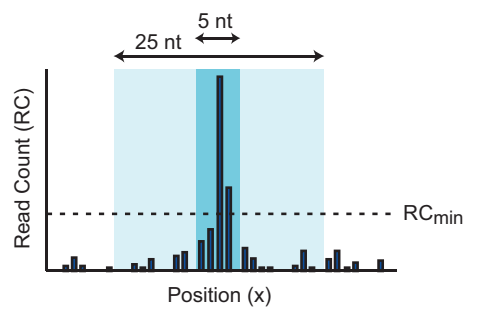

A peak at position $x$ is called a TSS if:

Read count ( $R C$ ) at position $x, R C_{x} \geq R C_{\text {min }}$

$R C_{x}$ is the largest value from $R C_{x-2}$ to $R C_{x+2}$

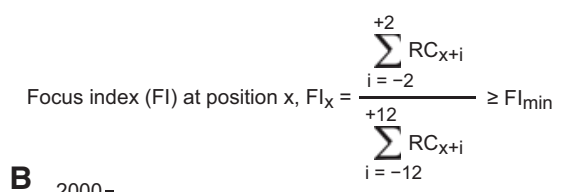

FocusTSS Position Relative to RefSeq TSS

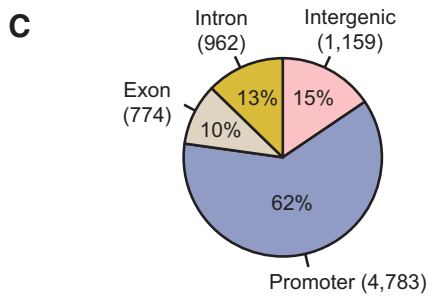

Figure 1. Identification of focused TSSs in $5^{\prime}$-GRO-seq data with FocusTSS. (A) The peak-calling scheme in FocusTSS is based on the properties of the transcription PIC. In the PIC, the polymerase is able to initiate transcription in a window of $\sim 5 \mathrm{nt}$. Thus, FocusTSS selects peaks based on the concentration of reads in a 5-nt window relative to the total reads in a larger 25-nt window. The formula used for peak calling is shown with a visual representation of the parameters. In our data from MCF-7 cells, we typically used a $\mathrm{RC}_{\min }$ of 20 (approximately one read per million) and a $\mathrm{FI}_{\min }$ of 0.67 . (B) FocusTSS peaks are generally close to annotated RefSeq TSSs. FocusTSS peaks (7678) were called with $\mathrm{RC}_{\min }$ of 20 and $\mathrm{FI}_{\min }$ of 0.67 , and the peak count was calculated for each bin within the indicated range of distances to the closest annotated TSS. (C) The majority of FocusTSS peaks is located in promoter regions. The 7678 FocusTSS peaks were classified according to their location in genomic elements. Most TSSs were located near or within annotated genes. Promoters were defined as the region from $-1000 \mathrm{nt}$ to $+100 \mathrm{nt}$ relative to the closest annotated TSS. The numbers of TSSs in each group are shown in parentheses. peaks that have at least a minimal read count $\left(\mathrm{RC}_{\min }\right)$ and are larger than other peaks in their immediate $( \pm 2-n t)$ vicinity. For each peak, it then determines whether the combined reads in a narrow 5-nt window centered on that peak are at least a minimal proportion (the minimal focus index $\left.\left[\mathrm{FI}_{\mathrm{min}}\right]\right)$ of the combined reads in a wider 25nt window that is centered on that peak. The FI reflects the extent to which transcription is focused at a single PIC. Examples of peaks with different FI values are in Supplemental Figure S1.

Hence, FocusTSS identifies isolated and focused TSSs that appear to derive from a specifically positioned PIC. For the purposes of this study, which is the analysis of the human Inr sequence, it is useful to have clearly separated and defined TSSs. For other applications, it is possible to vary parameters such as the window sizes, $\mathrm{RC}_{\text {min, }}$ and $\mathrm{FI}_{\mathrm{min}}$.

In our analysis of the human TSS data, we selected FocusTSS peaks with $\mathrm{RC}_{\text {min }}$ of 20 (approximately one read per million) and $\mathrm{FI}_{\text {min }}$ of 0.67 . With these criteria, the two independent 5'-GRO-seq data sets yielded 7678 shared peaks with similar properties (Supplemental Fig. S2). The 7678 FocusTSS peaks are found mainly near RefSeq-annotated TSSs for protein-coding and noncoding genes (Fig. 1B). Most (75\%; 5753 out of 7678 ) of the FocusTSS peaks are within $1 \mathrm{~kb}$ of a RefSeq TSS. In addition, the FocusTSS peaks are predominantly located in promoter regions (from -1000 to +100 relative to the RefSeq TSS) (Fig. 1C). (Because the 5'-GRO-seq method detects nascent transcripts, many of the nonpromoter TSS may be associated with short-lived species such as enhancer RNAs [eRNAs].) Hence, by the use of 5'-GROseq in conjunction with FocusTSS, we generated a data set of thousands of human focused TSSs that could be used for the analysis of core promoters.

\section{A new Inr consensus is frequently used in focused human promoters}

To identify overrepresented sequences in the immediate vicinity of the TSS, we analyzed our focused TSS data set with the HOMER motif discovery tool (Heinz et al. 2010). This yielded an Inr-like sequence (motif 1) (the frequency matrix is shown in Supplemental Fig. S3), the TCT motif (motif 2) (Parry et al. 2010; Wang et al. 2014), and two other sequences (Fig. 2A). The Inr-like sequence is the most abundant sequence in the vicinity of the TSS and has the consensus of $\mathrm{BBCA}_{+1} \mathrm{BW}$ (where $\mathrm{B}=\mathrm{C} / \mathrm{G} / \mathrm{T}$ and $\mathrm{W}=\mathrm{A} / \mathrm{T}$ ) from -3 to +3 relative to the +1 TSS (Fig. $2 \mathrm{~B})$. Given the prevalence of this sequence as well as its resemblance to various versions of the Inr in Drosophila and humans (Fig. 2C), it appears that $\mathrm{BBCA}_{+1} \mathrm{BW}$ is the consensus of the human Inr in focused promoters.

We further tested the range of conditions under which this consensus might be observed. To this end, we found that variation of $\mathrm{RC}_{\mathrm{min}}$ from 10 to 50 and $\mathrm{FI}_{\text {min }}$ from 0.50 to 0.75 resulted in $\mathrm{BBCA}_{+1} \mathrm{BW}$ (Supplemental Fig. S4A). In addition, we performed FocusTSS and HOMER analyses of $5^{\prime}$-GRO-seq or GRO-cap data sets from three other human cell lines (HeLa, GM12878, and K562) and obtained the same $\mathrm{BBCA}_{+1} \mathrm{BW}$ consensus (Supplemental Fig. S4B). Thus, the $\mathrm{BBCA}_{+1} \mathrm{BW}$ Inr consensus is widely observed in different conditions and cells.

Out of the 7678 focused TSS peaks in our MCF-7 data set, there are 3071 peaks $(40 \%)$ with a perfect match to 
A

\begin{tabular}{|c|c|c|c|}
\hline Motif & Sequence & $P$-value & Coverage \\
\hline & $\vec{\Gamma}$ & & \\
\hline $\begin{array}{c}1 \\
(\ln )\end{array}$ & 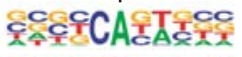 & $1 \mathrm{e}-851$ & $65.3 \%$ \\
\hline$\stackrel{2}{2}$ & TCTTICTITTC & $1 e-17$ & $1.1 \%$ \\
\hline 3 & TCCCAAAGATG & $1 e-14$ & $0.7 \%$ \\
\hline 4 & 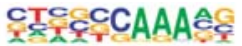 & $1 e-12$ & $1.2 \%$ \\
\hline
\end{tabular}

B

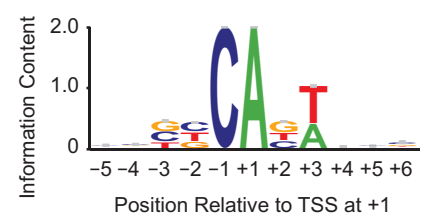

C

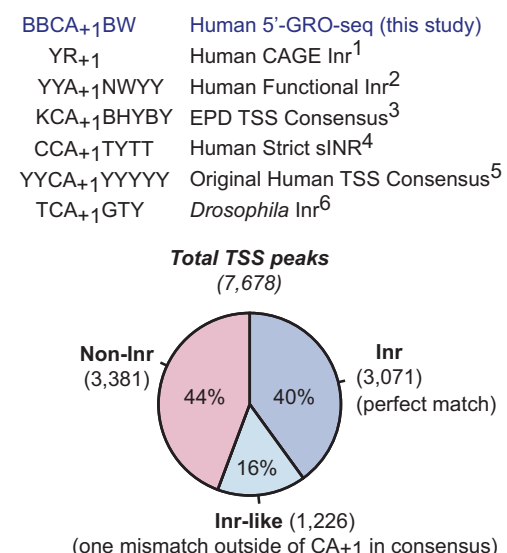

Figure 2. The $\mathrm{BBCA}_{+1} \mathrm{BW}$ consensus for the human initiator (Inr) is present in a majority of focused TSSs. (A) The Inr is the most abundant overrepresented sequence near the TSS. Motif discovery analysis of the -5 to +6 region (relative to the +1 TSS) was performed with 7678 focused TSSs in MCF-7 cells. The prevalence (coverage) and $P$-values of the top four sequence motifs are shown. Motif $1\left(\mathrm{BBCA}_{+1} \mathrm{BW}\right.$, where $\mathrm{B}=\mathrm{C} / \mathrm{G} / \mathrm{T}$ and $\mathrm{W}=\mathrm{A} / \mathrm{T}$ ) is the Inr, and motif 2 is the TCT motif (Parry et al. 2010). The arrow indicates the position of the TSS. (B) Sequence logo of the human Inr at focused TSSs. The sequences of the 3071 FocusTSS peaks with a perfect match to $\mathrm{BBCA}_{+1} \mathrm{BW}$ were used to generate the logo. $(C)$ Comparison of the new Inr consensus $\left(\mathrm{BBCA}_{+1} \mathrm{BW}\right)$ with some previously described Inr consensus sequences. (1) Human genome-wide CAGE (Carninci et al. 2006; Frith et al. 2008). (2) Functional consensus based on mutational analysis of the human Inr (Javahery et al. 1994; Lo and Smale 1996). (3) Single-nucleotide representation of position-weight matrix of the TSS consensus based on the Eukaryotic Promoter Database (EPD) (Bucher 1990). (4) A rare "strict Inr" in humans (Yarden et al. 2009). (5) The original consensus of the human Inr (Corden et al. 1980). (6) The Inr consensus in Drosophila (Ohler et al. 2002; FitzGerald et al. 2006). (D) The BBCA ${ }_{+1}$ BW Inr occurs frequently in focused promoters in humans. FocusTSS peaks were divided into three groups: perfect match (Inr), one mismatch outside of the central CA $\mathrm{CA}_{+1}$ (Inr-like), and all other sequences (nonInr). The number of TSSs in each group are shown in parentheses.

the $\mathrm{BBCA}_{+1} \mathrm{BW}$ Inr consensus and 1226 Inr-like peaks $(16 \%)$ that have only one mismatch outside of the central $\mathrm{CA}_{+1}$ in the consensus (Fig. 2D). Hence, the new Inr consensus is frequently observed in human promoters.

Moreover, the BBCABW sequence is strongly enriched at the +1 position of the FocusTSS peaks and is otherwise distributed randomly (Supplemental Fig. S5). This is consistent with the model that the Inr does not usually function by itself but rather acts in conjunction with other sequence motifs to give a fully active core promoter.

We wondered whether the TATA box or TATA-like sequences are enriched or depleted in promoters with Inr or
Inr-like motifs. To address this question, we examined the frequency of occurrence of either a consensus TATA box (TATAAR, as identified by HOMER, from -33 to -23 relative to the +1 TSS) or a degenerate TATA-like sequence (WWWW from -33 to -23 relative to the +1 TSS, where $\mathrm{W}=\mathrm{A} / \mathrm{T})$ in the Inr, Inr-like, or non-Inr promoters shown in Figure 2D. This analysis revealed that both consensus and degenerate TATA sequences were less common in Inr and Inr-like promoters than in non-Inr promoters (Supplemental Fig. S6A). For instance, the degenerate TATAlike sequence was observed in $\sim 21 \%$ and $23 \%$ of the Inr and Inr-like promoters, respectively, relative to $\sim 35 \%$ in non-Inr promoters (Supplemental Fig. S6A). It is possible that promoters with an Inr are less dependent on a TATA box and vice versa.

We also examined whether the consensus $\mathrm{BBCA}_{+1} \mathrm{BW}$ Inr is preferentially found within CpG islands. Approximately $60 \%$ of focused TSSs are found in CpG islands, but there is no apparent enrichment or depletion of BBCA $_{+1}$ BW Inr TSSs or Inr-like TSSs in CpG islands (Supplemental Fig. S6B). In contrast, focused TSSs that are associated with TATA-like sequences are depleted in CpG islands (Supplemental Fig. S6B).

As seen in Figure $2 \mathrm{C}$, the new $\mathrm{BBCA}_{+1} \mathrm{BW}$ Inr consensus is distinct from other versions of the human Inr. The widely used functional Inr consensus (YYA ${ }_{+1} \mathrm{NWYY)}$ (Javahery et al. 1994; Lo and Smale 1996) was based on the mutational analysis of the Inr. Another commonly used version of the human Inr $\left(\mathrm{YR}_{+1}\right)$ was obtained from genome-wide CAGE data (Carninci et al. 2006; Frith et al. 2008). The differences between the $\mathrm{YR}_{+1}$ consensus and the $\mathrm{BBCA}_{+1} \mathrm{BW}$ consensus may be due in part to the analysis of steadystate transcripts in the CAGE experiments and nascent transcripts in the 5'-GRO-seq and GRO-cap experiments. Another potential factor is the use of FocusTSS to identify focused start sites. Notably, we observed that TSSs with higher FI values are enriched for the $\mathrm{BBCA}_{+1} \mathrm{BW}$ Inr relative to TSSs with lower FI values (Supplemental Fig. S7A,B). Likewise, promoters with a perfect match to the $\mathrm{BBCA}_{+1} \mathrm{BW}$ Inr have higher FI values than promoters that do not contain a perfect match to the motif (Supplemental Fig. S7C). Thus, the selection of focused TSSs with FocusTSS enriches for promoters with the $\mathrm{BBCA}_{+1}$ BW Inr motif.

\section{Variants of the degenerate $B B C A_{+1} B W$ hexanucleotide at focused TSSS}

We next considered the possibility that some of the 54 variants of the $\mathrm{BBCA}_{+1} \mathrm{BW}$ consensus are overrepresented or underrepresented at promoters. To address this issue, we determined the frequency of occurrence of each of the 4096 possible hexanucleotide sequences from -3 to +3 (relative to the +1 TSS) in our data set of 7678 TSSs. This revealed that 46 of the 51 most abundant hexanucleotides are a perfect match to the $\mathrm{BBCA}_{+1} \mathrm{BW}$ consensus (Fig. 3A; Supplemental Fig. S8). Notably, there is not a specific subset of variants that is highly overrepresented. However, there is some underrepresentation of $\mathrm{BBCA}_{+1}$ TA and TGCA $_{+1} \mathrm{BW}$ sequences (Supplemental Fig. S8). Thus, nearly all of the 54 variants of the BBCA $_{+1} \mathrm{BW}$ Inr are among the most commonly used hexanucleotides at focused TSSs.

For comparison, we carried out the same analysis with the 32 variants of the functional Inr consensus 
A

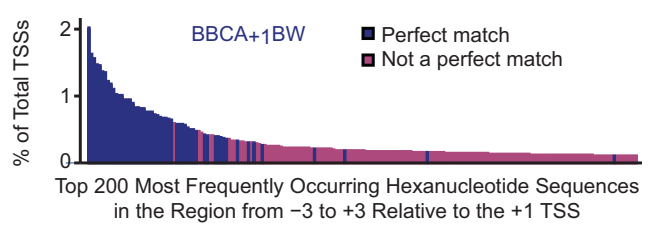

B

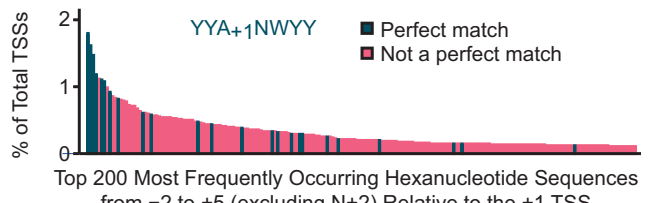

Figure 3. The $\mathrm{BBCA}_{+1} \mathrm{BW}$ Inr consensus generally represents the most frequently occurring sequences at the TSS. (A) Distribution of $\mathrm{BBCA}_{+1} \mathrm{BW}$ Inr sequences among the most frequently occurring hexanucleotides in the region from -3 to +3 relative to the +1 TSS. The plot shows the frequencies (percentage of total TSSs) of the top 200 (out of a possible 4096) occurring hexanucleotide sequences that are either a perfect match (blue) or not a perfect match (red) to the $\mathrm{BBCA}_{+1} \mathrm{BW}$ Inr consensus. All 54 versions of $\mathrm{BBCA}_{+1} \mathrm{BW}$ are in the top 200 sequences. The less frequently occurring perfect match outliers $\left(\leq 0.2 \%\right.$ frequency) are variants with the $\mathrm{BBCA}_{+1} \mathrm{TA}$ sequence (Supplemental Fig. S8). (B) The YYA ${ }_{+1}$ NWYY functional Inr consensus is somewhat broadly distributed among the most commonly occurring hexanucleotide sequences from -2 to +5 (excluding the random $N_{+2}$ ). The plot includes 24 out of the 32 variants of the $\mathrm{YYA}_{+1-}$. NWYY consensus.

(YYA ${ }_{+1}$ NWYY), which has six nonrandom positions from -2 to +5 relative to the +1 TSS (Fig. 3B; Supplemental Fig. S9). This revealed that eight of the 12 most common sequences are a match to the functional Inr; however, the other 24 variants of this consensus are not concentrated among the most commonly occurring sequences. Therefore, although the functional Inr consensus, which was elucidated $>20$ years ago, is an excellent representation of the Inr, the emergence of new technologies has now allowed the determination of the $\mathrm{BBCA}_{+1} \mathrm{BW}$ Inr, which is strongly represented at the genome-wide level among the most commonly occurring focused TSSs.

\section{Functional analysis of the $B B C A_{+1} B W$ sequences in the basal transcription process}

Next, we investigated the function of the $\mathrm{BBCA}_{+1} \mathrm{BW}$ Inr by in vitro transcription analysis of human core promoters in their natural context from -50 to +51 relative to the +1 TSS. In the first set of experiments, we examined the PMAIP1 and TFRC promoters, both of which contain a consensus BBCA $_{+1} \mathrm{BW}$ Inr. We tested a series of singlenucleotide substitution mutations for each position from -5 to +5 . Outside of the Inr (positions $-5,-4,+4$, and +5 ), we used transition mutations, whereas inside the Inr, we mutated the nucleotides to nonconsensus bases (Fig. 4A; Supplemental Fig. S10).

These studies indicated that the sequences from -1 to +3 , particularly the +1 and +3 positions, are important for core promoter activity. Moreover, we observed that $\mathrm{CA}_{+1}$, as in the $\mathrm{BBCA}_{+1} \mathrm{BW}$ consensus, mediates higher levels of transcription than $\mathrm{CG}_{+1}$ or $\mathrm{TA}_{+1}$, which match the $\mathrm{YR}_{+1}$ consensus. In addition, $\mathrm{B}_{-3}$ and $\mathrm{B}_{-2}$ (where $\mathrm{B}=$ not A) appear to contribute to focused initiation at $A_{+1}$, as we observed increased levels of transcription initiation at -3 and -2 when those positions are mutated to $\mathrm{A}$ (Fig. 4A; Supplemental Figs. S10, S11). Hence, single-nucleotide mutations that disrupt the $\mathrm{BBCA}_{+1} \mathrm{BW}$ consensus result in a reduction or an alteration of the activity of the core promoter. In contrast, mutations outside of the $\mathrm{BBCA}_{+1} \mathrm{BW}$ Inr consensus had little effect on core promoter function (Fig. 4A; Supplemental Fig. S10).

We additionally tested the effect of mutation of nonconsensus Inr sequences to the consensus sequence. To this end, we selected 12 naturally occurring core promoters that contain a single mismatch to the $\mathrm{BBCA}_{+1} \mathrm{BW}$ consensus at positions ranging from -3 to +3 and then generated single-nucleotide substitutions that convert the nonconsensus sequences to the $\mathrm{BBCA}_{+1} \mathrm{BW}$ Inr consensus (Fig. 4B). These experiments revealed that conversion of the nonconsensus sequences to the Inr consensus generally led to an increase in transcriptional activity, with the largest effects observed at the +1 and +3 positions.

Altogether, the mutational analyses indicate that transcription initiates optimally from the $\mathrm{BBCA}_{+1} \mathrm{BW}$ Inr consensus and that the region from -1 to +3 is most important for the efficiency of transcription. These results reflect the nucleotide distributions that were observed in the Inr region (Fig. 2; Supplemental Fig. 3) and are consistent with the findings of Smale and colleagues (Javahery et al. 1994; Lo and Smale 1996) in their analysis of the functional Inr consensus. In some promoter contexts, the lack of an A nucleotide at positions -2 and -3 appears to suppress transcription initiation at those sites and thus support more focused transcription from the +1 TSS. It is also notable that $\mathrm{CA}_{+1}$ more specifically reflects the active Inr element than the more general $\mathrm{YR}_{+1}$ consensus.

It can further be seen that $\mathrm{C}_{-1}$ and $\mathrm{A}_{+1}$ are more prominent in the Inr consensus than $\mathrm{W}_{+3}$, whereas $\mathrm{A}_{+1}$ and $\mathrm{W}_{+3}$ are more important for transcriptional activity than $\mathrm{C}_{-1}$. In addition, all of the 40 most frequently occurring hexanucleotides at the Inr region include $\mathrm{C}_{-1}, \mathrm{~A}_{+1}$, and $\mathrm{W}_{+3}$ (Supplemental Fig. S8). These findings collectively suggest that there is an additional constraint for the use of $\mathrm{C}_{-1}$ that extends beyond its role in contributing to promoter strength. As an example, such a constraint might be the need to avoid inadvertent binding by a sequencespecific factor with a related and/or overlapping recognition sequence.

\section{The human Inr, a distinct and abundant element that is precisely positioned at focused TSSS}

In this study, we identified and characterized the $\mathrm{BBCA}_{+1} \mathrm{BW}$ Inr consensus sequence, which is positioned precisely at more than half of focused human TSSs (Fig. $2)$. Of the 54 variants of this consensus, none are highly overrepresented; there is, however, some underrepresentation of $\mathrm{BBCA}_{+1} \mathrm{TA}$ and $\mathrm{TGCA}_{+1} \mathrm{BW}$ sequences (Fig. 3; Supplemental Fig. S8). Moreover, the TATA box and TATA-like sequences are less common in $\mathrm{BBCA}_{+1} \mathrm{BW}$ Inr and Inr-like promoters than in non-Inr promoters (Supplemental Fig. S6).

The articulation of the Inr element is essential for the understanding of the mechanisms of transcription in humans. This new consensus can now be used as a foundation for the analysis of the other sequences and associated factors that regulate gene activity. 
A

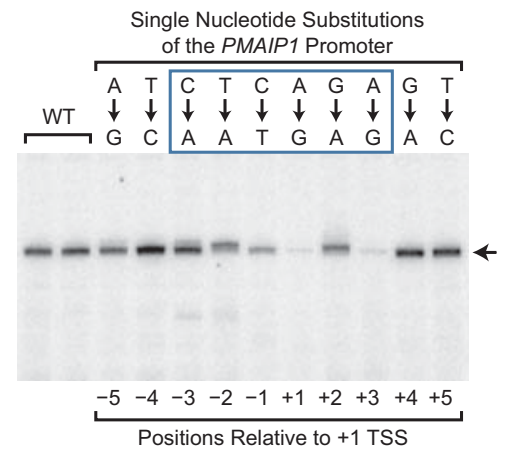

B

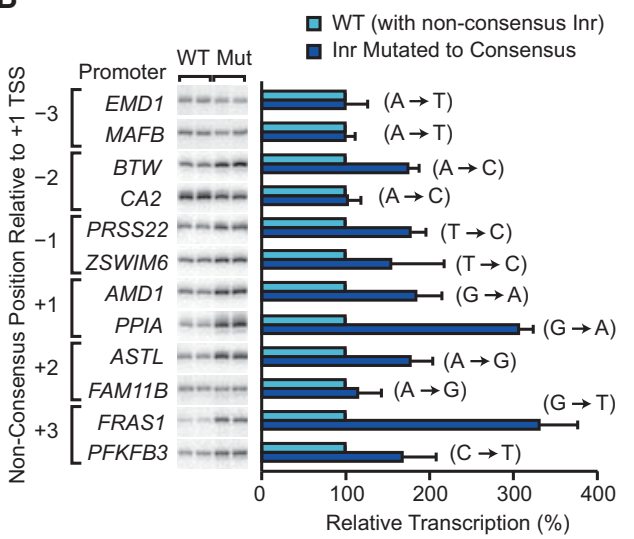

Figure 4. The $\mathrm{BBCA}_{+1} \mathrm{BW}$ Inr sequence is essential for efficient and accurate transcription initiation. The core promoter regions from -50 to +51 relative to the +1 TSS (for DNA sequences, see Supplemental Fig. S13) of the indicated human genes were used in these experiments. (A) Alterations in the Inr sequence impair transcription strength and start site selection. The consensus Inr sequence in the PMAIP1 promoter was mutated by using the indicated single-nucleotide substitutions. The wild-type (WT) and mutant constructs were subjected to in vitro transcription and primer extension analysis. Mutations in the $\mathrm{BBCA}_{+1} \mathrm{BW}$ Inr are inside the blue box. The horizontal arrow indicates the +1 TSS. Quantitation of the transcription levels from at least four independent experiments is shown in Supplemental Figure S10A. (B) Mutation of nonconsensus Inr sequences to the consensus generally results in higher levels of transcription. Nonconsensus wild-type promoters (WT) or mutant promoters that were altered at a single nucleotide to match the consensus (Mut) were subjected to in vitro transcription analysis. The single-nucleotide substitutions are indicated in parentheses. The autoradiograms show representative results, and the quantitative data from three or more experiments are shown as the mean (relative to wild type $) \pm S D$.

Importantly, it should be noted that this study has been restricted to the analysis of focused TSSs, which have a clearly isolated site (or narrow 5-nt region) at which transcription initiates. The analysis of focused TSSs has minimized ambiguity with regard to the sequences that direct transcription and has thus facilitated the elucidation of the Inr consensus. In addition, our MCF-7 data set yielded 7678 focused TSSs (Figs. 1, 2), which represent thousands of protein-coding genes and noncoding transcripts. Nevertheless, our analysis of focused promoters does exclude nonfocused promoters (also known as dispersed or broad promoters). Some nonfocused promoters may be tandemly arranged focused core promoters, whereas others may direct dispersed transcription by an entirely different mechanism.

At a practical level, we also considered the merits of a slightly simplified $\mathrm{BCA}_{+1} \mathrm{BW}$ Inr consensus. The exclu- sion of $\mathrm{B}_{-3}$ from the consensus was considered because the $\mathrm{B}_{-3}$ position exhibits the lowest amount of sequence conservation relative to the other positions (e.g., see Supplemental Fig. S4A), and mutation of $\mathrm{B}_{-3}$ has little effect on the overall strength of transcription (Fig. 4; Supplemental Fig. S10). We therefore carried out an analysis of the $\mathrm{BCA}_{+1} \mathrm{BW}$ sequence (Supplemental Fig. S12). This revealed that $45 \%$ of TSSs contain a perfect match to $\mathrm{BCA}_{+1} \mathrm{BW}$ and that an additional $13 \%$ of TSSs contain only a single mismatch to $\mathrm{BCA}_{+1} \mathrm{BW}$ outside of the central $\mathrm{CA}_{+1}$ dinucleotide. Moreover, the 18 variants of the $\mathrm{BCA}_{+1} \mathrm{BW}$ sequence include the 17 most frequently occurring pentanucleotide sequences at focused TSSs, and the overrepresentation of pentanucleotides that perfectly match $\mathrm{BCA}_{+1} \mathrm{BW}$ is striking (Supplemental Fig. S12C,D). Thus, the simplified $\mathrm{BCA}_{+1} \mathrm{BW}$ sequence is an excellent version of the human Inr.

In conclusion, the $\mathrm{BBCA}_{+1} \mathrm{BW}$ Inr and Inr-like sequences (with only one mismatch outside of $\mathrm{CA}_{+1}$ ) are found at precisely the same location in more than half of focused human TSSs (Fig. 2D; Supplemental Figs. 10D, 11B) and are much more abundant than the TATA box or TATAlike sequences (Supplemental Fig. S6). This revised Inr consensus should serve as a useful and reliable beacon for the study of transcription in humans.

\section{Materials and methods}

\section{5'-GRO-seq}

Two 5'-GRO-seq experiments were carried out with MCF-7 cells essentially as described in Duttke et al. (2015) and Hetzel et al. (2016). The detailed procedure is provided in the Supplemental Material. The 5'-GRO-seq data are available from Gene Expression Omnibus (GEO; accession number, GSE90035).

\section{FocusTSS}

FocusTSS is a Python program (Focus_TSS.py) and is available in the Supplemental Material. The design and use of FocusTSS is described in Figure $1 \mathrm{~A}$ as well as in the Supplemental Material.

\section{In vitro transcription assays}

The plasmids used in the in vitro transcription assays were constructed by insertion of core promoter sequences $(-50$ to +51 relative to the TSS) in the $\mathrm{XbaI}$ and PstI sites of the pUC119T vector. Transcription reactions were performed essentially as described previously (Theisen et al. 2013). The specific reaction conditions are indicated in the Supplemental Material. All in vitro transcription experiments were performed independently at least three times to ensure reproducibility of the data.

\section{Acknowledgments}

We thank E. Peter Geiduschek, George Kassavetis, and Yuan-Liang Wang for critical reading of the manuscript, and Chris Benner for advice on the computational analysis. Contributions by Thomas Boulay, Scott Iwashita, and Timothy Bretz to earlier functional studies of the human Inr are also acknowledged. This work was supported by National Institutes of Health grants R01 GM041249, R21 HG008781, and R35 GM118060 to J.T.K.

\section{References}

Bucher P. 1990. Weight matrix descriptions of four eukaryotic RNA polymerase II promoter elements derived from 502 unrelated promoter sequences. J Mol Biol 212: 563-578. 
Butler JE, Kadonaga JT. 2001. Enhancer-promoter specificity mediated by DPE or TATA core promoter motifs. Genes Dev 15: 25152519.

Carninci P, Sandelin A, Lenhard B, Katayama S, Shimokawa K, Ponjavic J, Semple CAM, Taylor MS, Engström PG, Frith MC, et al. 2006. Genome-wide analysis of mammalian promoter architecture and evolution. Nat Genet 38: 626-635.

Chalkley GE, Verrijzer CP. 1999. DNA binding site selection by RNA polymerase II TAFs: a TAF(II)250-TAF(II)150 complex recognizes the initiator. $E M B O$ J 18: 4835-4485.

Corden J, Wasylyk B, Buchwalder A, Sassone-Corsi P, Kedinger C, Chambon P. 1980. Promoter sequences of eukaryotic protein-coding genes. Science 209: 1406-1414.

Core LJ, Martins AL, Danko CG, Waters CT, Siepel A, Lis JT. 2014. Analysis of nascent RNA identifies a unified architecture of initiation regions at mammalian promoters and enhancers. Nat Genet 46: $1311-1320$.

Danino YM, Even D, Ideses D, Juven-Gershon T. 2015. The core promoter: at the heart of gene expression. Biochim Biophys Acta 1849: 1116-1131.

Duttke SHC, Doolittle RF, Wang YL, Kadonaga JT. 2014. TRF2 and the evolution of the bilateria. Genes Dev. 28: 2071-2076.

Duttke SHC, Lacadie SA, Ibrahim MM, Glass CK, Corcoran DL, Benner C, Heinz S, Kadonaga JT, Ohler U. 2015. Human promoters are intrinsically directional. Mol Cell 57: 674-684.

FitzGerald PC, Sturgill D, Shyakhtenko A, Oliver B, Vinson C. 2006. Comparative genomics of Drosophila and human core promoters. Genome Biol 7: R53.

Frith MC, Valen E, Krogh A, Hayashizaki Y, Carninci P, Sandelin A. 2008. A code for transcription initiation in mammalian genomes. Genome Res 18: 1-12.

Gershenzon NI, Ioshikhes IP. 2005. Synergy of human Pol II core promoter elements revealed by statistical sequence analysis. Bioinformatics 21: $1295-1300$.

Goodrich JA, Tjian R. 2010. Unexpected roles for core promoter recognition factors in cell-type-specific transcription and gene regulation. Nat Rev Genet 11: 549-558.

Heinz S, Benner C, Spann N, Bertolino E, Lin YC, Laslo P, Cheng IX, Murre C, Singh H, Glass CK. 2010. Simple combinations of lineage-determining transcription factors prime cis-regulatory elements required for macrophage and B cell identities. Mol Cell 38: 576589.

Hetzel J, Duttke SH, Benner C, Chory J. 2016. Nascent RNA sequencing reveals distinct features in plant transcription. Proc Natl Acad Sci 113: $12316-12321$.

Javahery R, Khachi A, Lo K, Zenzie-Gregory B, Smale ST. 1994. DNA sequence requirements for transcriptional initiator activity in mammalian cells. Mol Cell Biol 14: 116-127.
Juven-Gershon T,Hsu JY, Kadonaga JT. 2008. Caudal, a key developmental regulator, is a DPE-specific transcription factor. Genes Dev 22: 2823-2830.

Kadonaga JT. 1990. Assembly and disassembly of the Drosophila RNA polymerase II complex during transcription. J Biol Chem 265: 2624-2631.

Kadonaga JT. 2012. Perspectives on the RNA polymerase II core promoter. Wiley Interdiscip Rev Dev Biol 1: 40-51.

Kaufmann J, Smale ST. 1994. Direct recognition of initiator elements by a component of the transcription factor IID complex. Genes Dev 8: 821-829.

Kruesi WS, Core LJ, Waters CT, Lis JT, Meyer BJ. 2013. Condensin controls recruitment of RNA polymerase II to achieve nematode X-chromosome dosage compensation. Elife 2: e00808.

Lam MTY, Cho H, Lesch HP, Gosselin D, Heinz S, Tanaka-Oishi Y, Benner C, Kaikkonen MU, Kim AS, Kosaka M, et al. 2013. Rev-Erbs repress macrophage gene expression by inhibiting enhancer-directed transcription. Nature 498: 511-515.

Lo K, Smale ST. 1996. Generality of a functional initiator consensus sequence. Gene 182: 13-22.

Ohler U, Liao G, Niemann H, Rubin GM. 2002. Computational analysis of core promoters in the Drosophila genome. Genome Biol 3: RESEARCH0087.

Parry TJ, Theisen JWM, Hsu JY, Wang YL, Corcoran DL, Eustice M, Ohler U, Kadonaga JT. 2010. The TCT motif, a key component of an RNA polymerase II transcription system for the translational machinery. Genes Dev 24: 2013-2018.

Purnell BA, Emanuel PA, Gilmour DS. 1994. TFIID sequence recognition of the initiator and sequences farther downstream in Drosophila class II genes. Genes Dev 8: 830-842.

Smale ST, Baltimore D. 1989. The 'initiator' as a transcription control element. Cell 57: 103-113.

Smale ST, Kadonaga JT. 2003. The RNA polymerase II core promoter. Annu Rev Biochem 72: 449-479.

Theisen JWM, Gucwa JS, Yusufzai T, Khuong MT, Kadonaga JT. 2013. Biochemical analysis of histone deacetylase-independent transcriptional repression by MeCP2. J Biol Chem 288: 7096-7104.

Verrijzer CP, Chen JL, Yokomori K, Tjian R. 1995. Binding of TAFs to core elements directs promoter selectivity by RNA polymerase II. Cell 81: 1115-1125.

Wang YL, Duttke SHC, Chen K, Johnston J, Kassavetis GA, Zeitlinger J, Kadonaga JT. 2014. TRF2, but not TBP, mediates the transcription of ribosomal protein genes. Genes Dev 28: 1550-1555.

Yang C, Bolotin E, Jiang T, Sladek FM, Martinez E. 2007. Prevalence of the initiator over the TATA box in human and yeast genes and identification of DNA motifs enriched in human TATA-less core promoters. Gene 389: 52-65.

Yarden G, Elfakess R, Gazit K, Dikstein R. 2009. Characterization of sINR, a strict version of the Initiator core promoter element. Nucleic Acids Res 37: 4234-4246. 


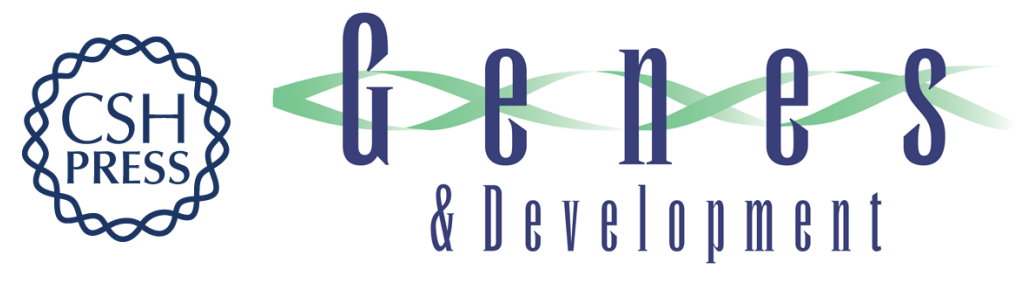

\section{The human initiator is a distinct and abundant element that is precisely positioned in focused core promoters}

Long Vo ngoc, California Jack Cassidy, Cassidy Yunjing Huang, et al.

Genes Dev. 2017, 31: originally published online January 20, 2017

Access the most recent version at doi:10.1101/gad.293837.116

\section{Supplemental http://genesdev.cshlp.org/content/suppl/2017/01/20/gad.293837.116.DC1 \\ Material}

Related Content Finding the start site: redefining the human initiator element Jennifer F. Kugel and James A. Goodrich

Genes Dev. January , 2017 31: 1-2

References This article cites 33 articles, 14 of which can be accessed free at:

http://genesdev.cshlp.org/content/31/1/6.full.html\#ref-list-1

Articles cited in:

http://genesdev.cshlp.org/content/31/1/6.full.html\#related-urls

Creative This article is distributed exclusively by Cold Spring Harbor Laboratory Press for the first Commons

License

six months after the full-issue publication date (see

http://genesdev.cshlp.org/site/misc/terms.xhtml). After six months, it is available under a Creative Commons License (Attribution-NonCommercial 4.0 International), as described at http://creativecommons.org/licenses/by-nc/4.0/.

Email Alerting

Receive free email alerts when new articles cite this article - sign up in the box at the top

Service right corner of the article or click here.

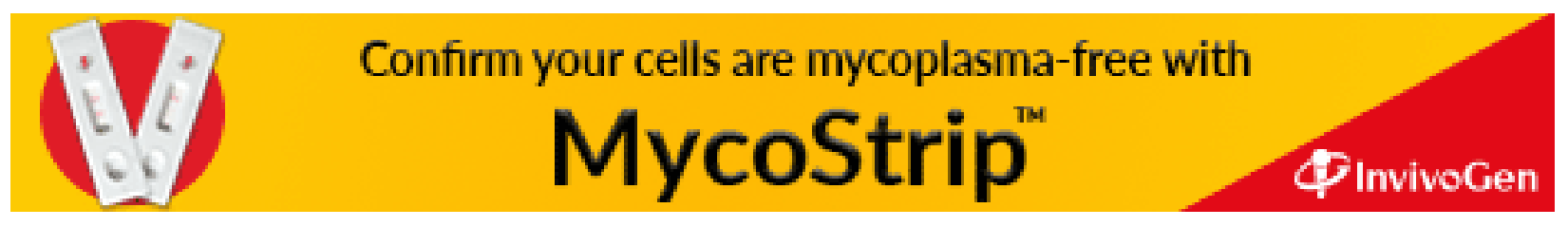

